\title{
Hans-Günter Thien
}

\section{Klassentheorien - Die letzten 50 Jahre $^{1}$}

„Es ist jedesmal das unmittelbare Verhältnis der Eigentümer der Produktionsbedingungen zu den unmittelbaren Produzenten - ein Verhältnis, dessen jedesmalige Form stets naturgemäß einer bestimmten Entwicklungsstufe der Art und Weise der Arbeit und daher ihrer gesellschaftlichen Produktivkraft entspricht -, worin wir das innerste Geheimnis, die verborgene Grundlage der ganzen gesellschaftlichen Konstruktion und daher auch der politischen Form des Souveränitäts- und Abhängigkeitsverhältnisses, kurz, der jedesmaligen Staatsform finden. Dies hindert nicht, daß dieselbe ökonomische Basis - dieselbe den Hauptbedingungen nach - durch zahllos verschiedne empirische Umstände, Naturbedingungen, Racenverhältnisse, von außen wirkende geschichtliche Einflüsse usw., unendliche Variationen und Abstufungen in der Erscheinung zeigen kann, die nur durch die Analyse dieser empirisch gegebnen Umstände zu begreifen sind (Marx 1969: 799f).

\section{Jenseits der nivellierten Mittelstandsgesellschaft}

Nehmen wir als Ausgangspunkt für die klassentheoretische Debatte in Deutschland die späten 1960er Jahre: Für die Mehrheit der Soziologen scheint die deutsche Gesellschaft kaum noch etwas mit dem zu tun zu haben, was Marx oder Weber als Klassengesellschaft charakterisierten. Eine solche mochte es im 19. Jahrhundert gegeben haben, aber inzwischen war doch viel Zeit vergangen, und hatte es nicht seitdem zweifellos wichtige Veränderungen in den Gesellschaftsverhältnissen gegeben, machte es überhaupt noch Sinn, von einem Kapitalismus in Deutschland oder anderswo zu sprechen? Waren nicht aus den Klassengegnern, die sich antagonistisch gegenüberstanden hatten, Sozialpartner geworden, deren Position sich zunehmend annäherte, was nicht zuletzt durch Verbesserungen mit der Sozialpolitik bewirkt wurde, die im schließlich durchgesetzten Wohlfahrtsstaat ihr Zentrum hatten? Auch wenn es heute skurril anmuten mag, Helmut Schelskys Bild von einer „nivellierten Mittelstandsgesellschaft“ bestimmte den soziologischen Mainstream und das Selbstverständnis vieler Deutscher. Dabei

1 Für die umsichtige redaktionelle Betreuung bedanke ich mich bei Peter Bescherer. 
wird auch der verlorene Krieg und der nötige Wiederaufbau eine Rolle gespielt haben, was eine besondere Form von Gemeinschaftlichkeit suggerieren mochte.

Aber spätestens mit dem Soziologentag im Jahre 1968 und dort insbesondere mit der Kontroverse zwischen Ralf Dahrendorf und Theodor W. Adorno über Industriegesellschaft oder Spätkapitalismus (Adorno 1969) wurde deutlich, dass diese behauptete Gemeinschaftlichkeit selbst in der Soziologie nur eine scheinbare war. Denn Adorno erdreistete sich in den Augen seiner Kontrahenten, trotz oder gerade wegen aller eingeräumten empirischen Modifikationen gegenüber den von Marx analysierten gesellschaftlichen Zusammenhängen nach wie vor von einer kapitalistischen Klassengesellschaft auszugehen und hierbei auch die Klassenfrage für zentral zu halten. Die Soziologie hatte ein Thema, das öffentliche Beachtung fand.

Nicht dass dies eine ausschließlich dem Anlass geschuldete vereinzelte $\mathrm{Zu}$ spitzung gewesen wäre. Horkheimer und Adorno und andere Mitglieder des Instituts für Sozialforschung hatten schon in den 1930er und 40er Jahren mit ihrer Konzeption des Organisierten Kapitalismus und des autoritären Charakters und insbesondere ihrer Faschismusanalyse wesentlich zur Analyse des historischen Prozesses beigetragen. Es ist geradezu frappierend, wie reflektiert etwa in den Reflexionen zur Klassentheorie (Adorno 1942) einerseits die Folgen kapitalistischer Vergesellschaftung im Blick sind und gleichzeitig einem versimpelten Marxismus begegnet wird, der zuweilen den Augenschein empirischer Faktizität auf seiner Seite zu haben scheint.

Aber auch nach 1945 haben sich die Kritischen Theoretiker kontinuierlich mit der konkreten kapitalistischen Verfasstheit der bundesrepublikanischen Gesellschaft auseinandergesetzt. In seiner Vorlesung zur Einleitung in die Soziologie (Adorno 1993), aus dem Jahre 1968, die geradezu exemplarisch hierfür stehen kann, kommt Adorno in immer neuen Anläufen und Einkreisungen auf die Klassenproblematik zu sprechen, die er als ,entscheidende Frage überhaupt für die Beurteilung der gegenwärtigen Gesellschaft“ (ebd.: 42) bezeichnet. Unter Rückbezug auf Marx weist er zugleich darauf hin, dass dessen Theorie eine im Kern historische sei und die gesellschaftlichen Veränderungen nicht nur zur Kenntnis zu nehmen, sondern auf den Begriff zu bringen sind. Das meint etwa die Marxschen Prognosen über eine Verelendung der proletarischen Massen oder eine generelle Zuspitzung des Klassengegensatzes. Adorno will nicht einfach nur die empirisch konstatierbaren Veränderungen einbeziehen, sondern angesichts solcher Veränderungen besteht er auf einer klassentheoretischen Behandlung des Sachverhaltes, dass Arbeitskräfte in kapitalistischen Gesellschaften einer Subjekt-Objekt-Problematik ausgesetzt sind. Zwar basiert die kapitalistische Klassengesellschaft letztlich auf ihren Handlungen (Arbeit), aber gleichzeitig ist sie den Resultaten ihrer eigenen Handlungen ausgeliefert; und schließlich 
gibt es die Möglichkeit, diese Abhängigkeit als potentielle Klassenindividuen eines potenziellen Handlungssubjekts, nennen wir es Proletariat, zu überwinden.

Gegenüber der Vorstellung vorgeblicher Automatismen zur befreienden Aktion gegenüber kapitalistischer Herrschaft sind Adorno und Horkheimer nicht zuletzt aufgrund ihrer Erfahrung mit dem nationalsozialistischen Faschismus gefeit; war es diesem nicht gelungen, auch eine erhebliche Zahl von Arbeiter_innen in seine „Volksgemeinschaft“ einzubinden? Und hatte sich nach 1945 nicht unübersehbar eine zunehmende Loslösung immer weiterer Kreise der Arbeiter_innenschaft von den Parolen und Zielen der Arbeiterbewegung ergeben. Das hatte sicher mit dem zu tun, was man als Errungenschaften des „Wirtschaftswunders" bezeichnet hat, womit nicht zuletzt die Verallgemeinerung der Kulturindustrie und ihre Implikationen gemeint sind. Herrschaft wandert geradezu in die Menschen ein; die Frage ist, in welchem Maße und ob und wie es Möglichkeiten gibt, dem zu begegnen.

Insofern bleibt bei Adorno offen, ob und wie die Menschen, die in unterschiedlicher Weise in den Reproduktionsprozess des Kapitals einbezogen sind, diese Herrschaft überwinden können, nein, wollen, um in gemeinsamer Aktion eine über die kapitalistische hinausgehende neue Gesellschaft zu schaffen. Es ist dies gleichzeitig eine Frage nach dem Stellenwert und der Zielsetzung des Tuns von Wissenschaftler_innen im Betreiben ihrer Wissenschaft. Angesichts der konkreten Verfasstheit westlicher Klassengesellschaften waren Adorno und mehr noch Horkheimer gegenüber den Möglichkeiten einer transformatorischen Praxis handelnder Gruppierungen äußerst skeptisch. Statt zu resignieren, wie es ihnen häufig nachgesagt wird, entwickelten sie jedoch eine besondere Form der Praxis, die Demirović (2004: 488) im Anschluss an Foucault als „Politik der Wahrheit" bezeichnet und versuchten damit, eine Traditionslinie kritischen Denkens herzustellen.

Es ist denn nur folgerichtig, dass auf dem angesprochenen Soziologentag auch eine Gruppe von Nachwuchswissenschaftlern aus dem Institut für Sozialforschung eine Konkretisierung über Herrschaft, Klassenverhältnisse und Schichtung (Bergmann u.a. 1969) vorlegte. In Anknüpfung an Marx und Adorno und insbesondere unter Akzentuierung der neuen Rolle des Staates als Interventionsstaat stellten die Autoren „die neueste Form der Institutionalisierung und staatlichen Kontrolle des traditionellen Klassenkonflikts“ (ebd.: 75) heraus. Mit anderer Begrifflichkeit meint dies in etwa das, was später in der Regulationstheorie als Fordismus und Klassenkompromiss gefasst wurde. Festgemacht wurde das, wie schon bei Adorno, v.a. an der korporatistischen Einbindung der Gewerkschaften in das Herrschaftsgefüge, wodurch wiederum Verbesserungen der Lage zumindest von Teilen der Arbeiter_innenschaft abgesichert wurden. Demgegenüber könnten andere Personengruppen aus diesem Klassenkompromiss ausgeschlossen bleiben 
und benachteiligt werden. Der Bezug zum Randgruppentheorem Marcuses liegt nahe. In gewissem Sinne handelt es sich um eine Vorwegnahme der Prekarisierungsthematik, die heute die Ungleich heits-Diskussion hierzulande über weite Strecken bestimmt. In Bezug auf das von der traditionellen Klassentheorie angenommene „immanente Potential historischer Veränderung “(ebd.:81) sind die Autoren skeptisch und verweisen auf die Notwendigkeit weiterer empirischer Analysen.

\section{Das Subjekt-Objekt-Dilemma}

Aber anscheinend war dies in Bezug auf die damalige politische Verfasstheit des linken Spektrums an den Universitäten viel zu verhalten für den gewünschten Umsturz und zu akademisch. Die Ambitionen der Autoren auf eine wissenschaftliche Karriere erschien dem linken Mainstream als dubios, was jene allerdings nicht daran hinderte, substanzielle Analysen zu verfassen (vgl.z.B. Brandt 1990), die noch heute zu beachten sind. In Anbetracht der imaginierten Situation eines unmittelbar bevorstehenden revolutionären Prozesses schien es nicht sinnvoll, die klassentheoretischen Fragen weiter zu verfolgen und empirisch zu vertiefen; anderes schien viel verheißungsvoller, nämlich der - so könnte man ein wenig vereinfachend sagen - nach der Abnabelung vom theoretischen Vater Adorno konsequent scheinende Weg zum Übervater Marx: eine oder besser: die Rekonstruktion der Marxschen Theorie und einer adäquaten politischen Praxis waren angesagt.

Genau hier setzte ein Teil der Studentenbewegung die Kritik an ihren Lehrern an. Beispielhaft hierfür kann der Adorno-Schüler Hans-Jürgen Krahl stehen. Mit einem revolutionären Impetus kritisiert er Adornos oder auch Habermas' Skepsis gegenüber den Möglichkeiten revolutionärer Praxis und insbesondere gegenüber den Möglichkeiten der Student_innen als Resignation und grundsätzliche Verkürzung. Dabei kann sich die Diagnose, noch nie zuvor sei die Klasse der Lohnabhängigen mehr Klasse an sich als für sich gewesen (vgl. Krahl 1971: 253), durchaus auf Adorno stützen. „Daraus aber schließen zu wollen, dass der in die gesellschaftliche Latenz verdrängte revolutionäre Klassenkampf nicht mehr aktualisierbar sei, wäre ebenso verfrüht wie anzunehmen, er könne weiterhin in seinen alten Formen sich vollziehen.“ (Ebd.)

Zwar gebe es einen „Bedürfnisfortschritt“, er bleibe aber in die Bahnen einer „kulturindustrielle(n) Regression von Kultur“ gebannt. Und angesichts der technologischen Veränderungen sei insbesondere die veränderte Rolle der (nicht nur) technischen Intelligenz herauszuarbeiten. Die ,revolutionstheoretisch entscheidenden Probleme der historischen Genesis des Klassenbewußtseins“ (ebd.: 343), 
denen sich Krahl gegenübersieht, führten in gewissem Sinne zurück in die 1920er Jahre, als die Problematik des Übergangs von der „Klasse an sich“ zu der „für sich “ insbesondere von Georg Lukács prominent thematisiert worden war. Hatte Marx hiermit auf eine grundlegende Problematik verwiesen, die sich allerdings in unterschiedlichen historischen Kontexten aufgrund der jeweiligen gesellschaftlichen und politischen Kräfteverhältnisse sehr unterschiedlich darstellte, so nahm Lukács sie als allgemeine auf, nämlich als Subjekt-Objekt-Konstellation, in der einzelne Proletarier wie Proletariat als Klasse sich befinden und die nur dialektisch gelöst werden könne: Einerseits als Einzelne dem Fetischismus der kapitalistischen Warengesellschaft ausgesetzt sei andererseits nur die Klasse in der Lage, diese aufzuheben, denn sie ist „das identische Subjekt-Objekt, das Subjekt der Tathandlung" (Lukács 1922/1967: 164). Aber das Proletariat bleibt gesichtslos, es bleibt Potenz, gebannt in die vorgegebenen Bahnen des Warentauschs und der Profitproduktion und -realisation, schließlich angewiesen auf eine Organisation, welche die gegebenen Widersprüche aufnimmt und entschlüsselt, eine Partei, aus der dann die Partei wurde. Damit aber macht Lukács „einen Sprung von dem einzelnen Proletarier zum Proletariat, das er sogleich als Substanz aufwerten muß. Die Dialektik von Allgemeinem und Besonderem, deren Mangel ein wesentlicher Grund für die Unmöglichkeit der Lösung der Antinomien des bürgerlichen Denkens war, wird zugunsten des Allgemeinen geschlichtet" (Negt/Kluge 1992: 123). Für den sich revolutionär verstehenden Teil der Student_innenbewegung der 1970er Jahre war die in den 20er Jahren gesuchte Lösung also eher eine Scheinlösung; sie war gleichzeitig eine neue Aufgabenstellung.

\section{Fünf Wege zum Klassenbewusstsein}

Aber wie sollte das praktisch unter den seinerzeit vorherrschenden gesellschaftlichen Bedingungen geschehen können? Wie sollte eine sich als revolutionäre Avantgarde verstehende Bewegung von hochqualifizierten Personen, wenn man will Bildungsbürgern, die in sich unterschiedliche Gruppierungen aufwies, sich mit jenen Massen verbinden, die man in der Regel als angepasst oder/und unpolitisch, als der kapitalistischen Konsumsphäre verhaftet ansah, und die eben nicht durch Proteste auf sich aufmerksam machten? - Eine schwierige und in sich widersprüchliche Ausgangskonstellation, die unterschiedliche Orientierungen und Handlungsvarianten eröffnete und aus sich heraussetzte. In aller hier gebotenen Kürze können die folgenden Wege oder Varianten skizziert werden: 


\subsection{Betriebsarbeit}

Ausgehend vom Wissen über das Wesen des Kapitalismus, und mochte es auch noch so begrenzt sein, und die „Mission der Arbeiterklasse“, und mochte sie auch noch so eingebildet sein, nahmen viele den „Weg in die Betriebe“ auf sich (vgl. zum Folgenden die dichte Analyse von Arps 2011). Die beteiligten Leitungsorganisationen, etwa der Kommunistische Bund Westdeutschlands (KBW), die Kommunistische Partei Deutschlands/Aufbauorganisation(KPD/AO), die Kommunistische Partei Deutschlands/Marxisten-Leninisten (KPD/ML) oder auch die DDR-nahe DKP gingen von der Notwendigkeit eines Klassenverrats aus, um, frei nach Mao Tse-Tung, wie ein Fisch im Wasser, im Betrieb und im Proletariat schwimmen zu können. Gleichzeitig behauptete man durch die Parteigründungen einen Führungsanspruch, da die in den Blick genommenen Proletarier von ihrer vorgeblichen historischen Mission noch gar nichts wussten oder eine solche gar ablehnten. Untereinander waren die Kader-Parteien sich in der Regel spinnefeind und der Aufenthalt in den Betrieben war mühsamer als gedacht. Die Kolleg_innen hatten ebenso wenig auf sie gewartet wie die Betriebsräte. Und auch die massiven Bekämpfungsmaßnahmen durch viele Gewerkschaften, die nicht selten in einen Gewerkschaftsausschluss mündeten, erschwerten das innerbetriebliche Handeln erheblich. Viele stiegen nach einiger Zeit wieder aus, andere lernten, wenigen erschloss sich schließlich eine betriebliche Karriere, die mitunter bis zum Vorsitz des Gesamtbetriebsrats eines großen Auto-Konzerns führen konnte.

Ähnliches gilt für kleinere Gruppierungen, die eher autonome Zielsetzungen im Betrieb zu realisieren suchten, wie der „Revolutionäre Kampf“ in Frankfurt/ Rüsselsheim, und sich an Konzepten des italienischen Operaismus orientierten. Auch wenn sie zeitweise einige Resonanz bei migrantischen Arbeitern gewinnen konnten, deren Situation sie als einzige im Blick hatten, blieben sie auf längere Sicht eher am Rande. Das wurde deutlich bei den insbesondere von migrantischen Arbeiterinnen und Arbeitern getragenen aufsehenerregenden Streiks bei Pierburg in Neuss oder bei Ford/Köln, bei denen diese auf sich selbst verwiesen blieben. Der geringe Rückhalt in der deutschen Arbeiter_innenschaft wurde rasch durch den Schulterschluss von Betriebsrat/Gewerkschaften und Betriebsleitung beendet (vgl. Bojadžijev 2012). Eine der wenigen oppositionellen Gruppierungen, die mit dieser Schwerpunktsetzung einer alternativen Betriebspolitik auf Dauer gestellt werden konnte, war die „plakat-Gruppe“ bei Mercedes-Benz (vgl. Grohmann/Sackstetter 1979), die noch immer dort tätig ist; Willi Hoss als einer ihrer Protagonisten, der aus der KPD gekommen war, sollte dann die Verbindung zu den neuen sozialen Bewegungen herstellen, indem er Gründungsmitglied und Bundestagsabgeordneter der „Grünen“ wurde, um diese dann später zu verlassen und sich Basisaktivitäten mit Indianern in Lateinamerika zuzuwenden (vgl. Hoss 2006). 
Die meisten autonomen Kader hatten den Betrieb da schon wieder verlassen, manche resignierten, andere hatten gelernt, wieder andere suchten weiter den Strand unter dem Pflaster, den nicht wenige in Landtagen oder im Bundestag fanden. Aber nur einem blieb es vorbehalten, durchzustarten und das Amt eines weltweit geschätzten Außenministers zu erklimmen; schließlich blieben der Weg in eine Beratungsagentur für Großkonzerne, ab und an eine Kolumne in ZEIT oder SZ und die Darstellung des Grandseigneurs der Grünen.

\subsection{Theoriearbeit}

In einer anderen Variante wurde die Aufgabenstellung in einer vertieften Aneignung der Marxschen Klassenanalyse gesehen. Eine der ersten gründlichen Aufarbeitungen wurde beispielsweise von Michael Mauke (1970) vorgelegt. Im Anschluss daran entstand eine Fülle weiterer Arbeiten, in denen die etwa schon in Marx' Grundrissen thematisierten Fragen einer Ausweitung und Veränderung des gesellschaftlichen Arbeitskörpers behandelt wurden, nämlich die Fragen nach der Verwendung von Resultaten der Wissenschaft in Produktion und Dienstleistung. Aber die Frage nach dem Stellenwert der Grundrisse (Marx 1974), der Resultate (Marx 1974) oder auch des Kapital für eine Klassenanalyse bleibt dennoch aktuell. Man könnte sagen, dass all die dort zu findenden expliziten Hinweise auf Klassen und Klassenkampf letztlich nur einen gesellschaftlichen Grundsachverhalt illustrieren, dem die Besitzer der Ware Arbeitskraft als Anhängsel des Kapitals und als in sich differenzierter Gesamtarbeitskörper ausgesetzt sind. Das gilt auch für die Aufschlüsselung der Kapitalseite in die unterschiedlichen Sorten von Kapitalisten bis hin zu ihren Administratoren (fungierendes, Kaufmanns-, Handelskapital). Es geht hier insgesamt um Personifikationen ökonomischer Verbältnisse, nicht um handelnde Personen und Personengruppen in ihrer historischen Vieldimensionalität. Nicht zufällig endet das Kapital mit einem Hinweis auf ein noch zu schreibendes Kapitel über Klassen. Ganz unbeschadet davon ist das Kapitalverhältnis und mit ihm das Klassenverhältnis als ein historisches zu denken und in seiner empirischen Verlaufsform als die vielen Kapitale und der konkrete Gesamtarbeitskörper in seiner inneren Differenziertheit jeweils zu erschließen.

Im Anschluss hieran entstand etwa durch marxistisch inspirierte industriesoziologische Forschungen sowohl ein sehr viel realistischeres Bild vom Bewusstsein von Arbeitern (vgl. Kudera u.a. 1979) als auch von deren interner Zusammensetzung, insbesondere in Gestalt der technisch-wissenschaftlichen Lohnarbeiter (Meschkat/Negt 1973; Beckenbach u.a. 1973). Eine herausragende Bedeutung gewann in der BRD dabei die Frage der gesellschaftlichen Verortung von Angestellten, die zwar schon von Siegfried Kracauer (1929) luzide behandelt worden war, gemeinhin aber zur Widerlegung einer marxistischen Klassen- 
theorie ins Gespräch gebracht wurde. Demgegenüber konnte die Besonderheit der Lage von angestellten Lohnarbeiter_innen und ihre Nähe zu Industriearbeitern in einer breit angelegten Studie von Ulf Kadritzke (1974) ebenso belegt werden wie ihre historischen Modifizierungen nicht zuletzt durch politische Interventionen. Das wurde in vielen weiteren Arbeiten belegt und durch eine ähnliche, aber interessenpolitisch völlig andere Entwicklung bei den cadres in Frankreich (vgl. Boltanski 1990) bestätigt (siehe zusammenfassend zur klassentheoretischen Begrenztheit Kadritzkes Nachruf auf die deutsche Kragenlinie, 1982). Als praktisch-politische Problematik etwa in der Gewerkschaftspolitik war „die“ Angestellten-Frage für eine Arbeiter_innenbewegung damit allerdings keineswegs gelöst.

Das Projekt Klassenanalyse versuchte in ähnlicher Weise eine stringente MarxInterpretation unter Aufnahme und Analyse der empirischen Veränderungen der Klassenstruktur zu entwickeln. Wie sehr hatte man in SOPO, PROKLA und später Sozialismus um die rechte Auslegung der Marxschen Schriften gerungen, sei es nun um den Begriff der Ware, die Reproduktionsschemata oder das Verhältnis von produktiver und unproduktiver Arbeit, mit denen man die Mystifikation des Waren- und Kapitalfetischs zu durchdringen versuchte, um zum Kern der Klassenkonstitution vorzudringen. Schließlich verbanden die Autoren den Kampf durch die drei blauen Bände mit der Einverleibung statistischer Materialien und legten voluminöse Materialien zur Klassenstruktur der BRD $(1973 ; 1974)$ vor. Das war durchaus informations- und lehrreich, aber die erhoffte wissenschaftliche Klärung von „Grundfragen revolutionärer Taktik“ (Bischoff) mochte sich letztendlich auch nach 1084 Seiten nicht einstellen; vielmehr wurde zur Erreichung dieses Ziel auf weiter zu gehende analytische Schritte verwiesen.

Vergleichbares gilt für das der DKP assoziierte Institut für Marxistische Studien und Forschungen (IMSF), das eine dreibändige Studie zur „Klassen- und Sozialstruktur der BRD 1950-1970“vorlegte und auf die Arbeiterklasse und ihre Aktionen hofften. Diese stellten sich im gewünschten Sinne allerdings nicht ein.

Demgegenüber verfolgten andere im Anschluss an die Arbeiten von Nicos Poulantzas in Politische Macht und gesellschaftliche Klassen (1974) und dann in Klassen im Kapitalismus - heute (1975) sowie im Anschluss an Althusser und Gramsci die eher klassisch marxistische Frage zum Verhältnis von Politik und Ökonomie im Staat und die Art der Einbindung der Klassen in ebendiesen.

Poulantzas differenziert zwischen Produktionsweise und Gesellschaftsformation und geht bei der Betrachtung einer vorherrschenden Produktionsweise vom grundsätzlichen Widerspruch zwischen Bourgeoisie und Arbeiterklasse aus, um zwischen Fraktionen innerhalb dieser Klassen und anderen gesellschaftlichen Kategorien zu differenzieren, die sich ihnen zuordnen oder zugeordnet werden. Historische Konjunkturen und Veränderungen des Herrschaftsgefüges werden 
getrennt betrachtet, wobei sich Poulantzas insbesondere auf die herrschenden Klassen bezieht, während die Arbeiterklasse eher vernachlässigt wird. Beim Kapital geht er von einem Gegensatz zwischen großem und mittlerem Kapital aus, was sich überschneidet, aber nicht deckungsgleich ist mit dem Unterschied zwischen national und international orientierten Einzelkapitalen. Das enthält angesichts der zunehmenden Globalisierung des Kapitals eine erhebliche Brisanz. Denn während man trotz der Imperialismustheorien von Lenin und Rosa Luxemburg letztlich immer mit einem methodologischen Nationalismus von nationalen Bourgeoisien ausgegangen war, stellte Poulantzas nun die Frage nach einer internationalen Bourgeoisie, der möglicherweise eine „Compradorenbourgeouisie“ und „innere Bourgeoisie“ subordiniert waren. Gab es also tatsächlich eine einzige "Atlantic Ruling Class“ (van der Pijl 1984) und in welcher Weise standen die nationalen Bourgeoisien (bzw. ihre Fraktionen) zu dieser, und wie drückte sich das in der politischen Praxis aus? Max Koch (2006: 133) betont zu recht die Bedeutung von Poulantzas, weist aber auch auf die Problematik einer empirischen Fundierung solcher Konzeptualisierungen hin.

Ähnliche Probleme betreffen auch die Untersuchungen von Joachim Hirsch (1995) und Hirsch/Roth (1986) über den zeitgenössischen Kapitalismus. Selbstverständlich diskutieren auch sie Veränderungen in den Klassenverhältnissen, stellen diese aber nicht ins Zentrum; die Art der Einbindung von Arbeiter_innen in das sich verändernde Herrschaftsgefüge wird zwar thematisiert, bleibt aber letztlich eigentümlich offen. Als unhintergehbar heben sie die Internationalisierung von Klassenbildungsprozessen hervor (vgl. inzwischen Hirsch/Wissel 2011; Wienold 2011).

\subsection{Geschichte und Erfahrung}

Andere wiederum versuchten, der Problematik durch eine historische Rekonstruktion der Arbeiterbewegung näher zu kommen, denn die gängigen ParteiSchriften aus der DDR genügten den neuen Ansprüchen ebenso wenig wie die der sozialdemokratischen Historiographie. Um nur drei Beispiele einer Vielzahl dann folgender Arbeiten zu nennen:

Oskar Negt und Alexander Kluge untersuchten in Öffentlichkeit und Erfahrung die „Erfahrungen und Interessen der proletarischen Klasse im weitesten Sinne" (1972:14) und versuchten dabei die immanenten Blockaden aufzuzeigen, die mit bürgerlicher Öffentlichkeit gegeben waren und durch eine proletarische Öffentlichkeit überwunden werden sollten. Hierin nahmen sie die von der Studentenbewegung gestellte Aufgabe einer (Neu)Bestimmung von Anknüpfungspunkten an die auch subjektive Betroffenheit von Arbeiter_innen von den sog. „Bewegungsgesetzen“ der kapitalistischen Produktionsweise auf. 
Erhard Lucas kam über seine Analyse der Ruhrkämpfe im Jahre 1920 zu Zwei Formen von Radikalismus in der deutschen Arbeiterbewegung, um schließlich das „Scheitern der deutschen Arbeiterbewegung" konstatieren zu müssen.

Karl-Heinz Roth wiederum suchte Die andere Arbeiterbewegung und rekonstruierte gegenüber den Partei-Organisationen eine Autonomie des Kampfes. Inspiriert durch den italienischen Operaismus, der den Kapital-LohnarbeitNexus umpolte, indem er letztendlich einfach die Arbeitermacht zum Movens der Veränderungen des Verhältnisses erklärte, denen das Kapital zu folgen habe, lieferte Roth einen lesenswerten Beitrag; strategisch bleibt er jedoch nostalgisch.

Insgesamt wird man sagen können, dass auf diesem Wege in einem schwierigen Forschungs- und Lernprozess wichtige Kenntnisse über die unterschiedlichen Lagen und Handlungen von Arbeiter_innen gewonnen wurden, wobei sich das politische Handeln eben gerade nicht aus einer objektiven Lage ergab, sondern Moment eines in sich vielfach gebrochenen und dynamischen Zusammenhangs war, der je neu zusammengesetzt wurde. Das gilt insbesondere für die in Deutschland besonders wichtige Phase des Nationalsozialismus, für die gegenüber den im akademischen Feld vorherrschenden Sichtweisen wie auch gegenüber parteihistoriografischen Ansätzen etwa von Tim Mason (1982) und Alf Lüdtke (1993) bis dahin wenig beachtete und notwendige Differenzierungen erschlossen wurden.

Einer der zentralen Begriffe für die Behandlung von Klassen und Handeln wurde Erfahrung. Hiermit stellte man sich innerhalb der zeitgenössischen marxistischen Diskussion über eine Struktur- bzw. handlungstheoretische Akzentuierung, wie sie zwischen Louis Althusser (1977) und E. P. Thompson (1968; 1980) geführt wurde, eindeutig auf die Seite Thompsons. Es war dann nur folgerichtig, dass Arbeiter_innengeschichte zunehmend als Alltagsgeschichte verstanden wurde. Über ähnliche Tendenzen etwa in der Soziologie hinausgehend mündete das hierzulande in die „Geschichtswerkstätten“, eine bundes- und schließlich europaweite Vernetzung von sich selbst als „Barfußhistoriker“ verstehenden Personen und Gruppierungen, die sich um Zeitschriftenprojekte wie die WerkstattGeschichte oder das History WorkshopJournal in Großbritannien scharten. Die Bezugnahme auf die Klassenthematik war anfangs zentral, wie etwa am dort verwendeten Begriff der "Arbeiterkulturen“ deutlich wird, mit dem das Feld historisch erschlossen wurde (vgl. zusammenfassend Thien 1998/2010, 122-151). Sie verlor jedoch an Bedeutung oder wurde ergänzt um die Geschlechterfrage, um schließlich nicht gänzlich zu fehlen (siehe WerkstattGeschichte (2005), aber gegenüber Themen der Neuen Sozialen Bewegungen an Bedeutung zu verlieren (siehe unten). 


\subsection{Postmarxismus - Übers Ziel hinaus}

Es mutet fast wie eine Ironie der Geschichte an, dass sich die, wenn man es einmal ganz abgekürzt sagt, Thompson-Anhänger einer erfahrungsgesättigten Klassengeschichtsschreibung an diesem Punkt mit ihren Kontrahenten, den Althusser-Vertretern einer Strukturtheorie, in ihrer Absage an die „Alte Soziale Bewegung“ trafen. Die Kritik von Althusser an Marx” „humanistischer Phase“ aufnehmend dehnten die heute als maßgebliche Vertreter des Poststrukturalismus geltenden Ernesto Laclau und Chantal Mouffe (1991) diese auf den ganzen Marx und insbesondere seine Klassentheorie aus. Mit ihrem „Postmarxismus“ konnten sie zwar einen bei marxistischen Theoretikern allzu häufig verbreiteten Klassenreduktionismus, der sich letztlich aus einem Ökonomismus und einem teleologischen Geschichtsdeterminismus ergibt, treffen. Ob die Marxsche Theorie, die bekanntlich in sich unterschiedliche Schichten aufweist, dadurch zureichend aufgenommen ist, sei dahingestellt. Ganz unabhängig davon bleibt die Frage, ob so die klassentheoretisch zentrale Verbindung von Struktur, Lage und Handeln in erweiterter Weise möglich wird. Denn statt einer inhaltlichen Vertiefung widmeten sich Laclau/Mouffe anschließend methodologischen und allgemein konzeptionellen Fragen etwa im Anschluss an Gramsci nach dem Stellenwert von Hegemonie. Auch das ist zweifellos wichtig, ist doch von diesem erstmals in der marxistischen Theorie das Problem der Einbindung von Arbeiter_innen in die kapitalistischen Herrschaftsformen insbesondere in Gestalt des Staates als ein zentrales artikuliert worden. Aber Gramsci bezieht sich dabei gleichwohl auf die Klassenproblematik, was Laclau/Moffe ihm wiederum vorwerfen. Aus diesem Grunde erscheint es mir symptomatisch bei ihnen, dass im Verlaufe der Zeit die Klassenfrage gegenüber Fragen der Demokratie in den Hintergrund gerückt wurde, womit dann die Neuen Sozialen Bewegungen für sie wichtiger wurden.

\subsection{Feminismus und neue soziale Frage}

Schließlich gab es quer zu diesen Versuchen, aber durchaus verbunden mit ihnen, die Neubegründung eines Feminismus, in dem zumindest anfangs auch die Klassenfrage intensiv diskutiert wurde. Denn allzu offensichtlich war die Randstellung von Frauen in der sich als revolutionär verstehenden Student_innenbewegung gewesen, allzu offensichtlich auch ihre weitgehende Ausblendung aus den exzessiven Theorie-Exegesen und ebenso offensichtlich die Benachteiligung von Frauen gegenüber ihren männlichen Kollegen in Produktion und Dienstleistung. Beginnend mit einer sukzessiven Trennung vom SDS, dem seine Frauenfeindlichkeit vorgeworfen wurde, entwickelten sich innerhalb kurzer Zeit verschiedene Stränge einer radikalen feministischen Bewegung, die bald eine große Bandbreite enthielt: Fragen nach Klasse Geschlecht (Beer 1987) stellten 
die besondere Art der Einbezogenheit von Frauen in den Produktions- und vor allem Reproduktionsprozess des Kapitals in den Mittelpunkt. Die Lage von Arbeiter_innen ist eben in sich differenziert (Becker-Schmidt/Knapp 1985), was in der Geschichte der Arbeiterbewegung selten beachtet worden war und auch in der aktuellen Gewerkschaftsbewegung ausgeblendet wurde. Andere behandelten die Spezifik von Hausarbeit und betonten im Unterschied zur in der marxistischen Diskussion gängigen Behandlung von produktiver und unproduktiver Arbeit im Sinne des Kapitals die gesellschaftliche Notwendigkeit der Arbeit zur individuellen Reproduktion; manche forderten Möglichkeiten für deren Entlohnung. Folgerichtig entstanden Arbeiten zu Familie und Klasse (Milhofer 1973) und historische Arbeiten zur Herausbildung eines weiblichen Proletariats (Friese 1991) und zu Proletarischen Familien (Rosenbaum1992). Aber solche Themen gerieten mit dem Siegeszug des Feminismus zunehmend ins Hintertreffen, während mit ihrer Benachteiligung im Beruf oder in Hinblick auf Abtreibungsfragen die politische Unterdrückung von Frauen an Bedeutung gewann, um schließlich auch die existenzielle Dimension eines „Frauseins“ einzubeziehen, über die im Anschluss an Simone de Beauvoir (1968) heftige Debatten geführt wurden, die konsequenterweise auch zur Thematisierung von „Männlichkeit“ führten. Eine Akademisierung des Diskussionsfeldes war unübersehbar. Im Anschluss daran artikulierten sich auch Homosexuelle und Lesben zunehmend als organisierte Gruppen eines sich autonom verstehenden alternativen Politikfeldes.

Im Laufe der Zeit war eine innere Separierung der einzelnen Diskussionsund Handlungsfelder voneinander nicht zu übersehen. Hier begannen sich die Konturen einer „neuen sozialen Frage“ abzuzeichnen, die im Begriff war, die „alte soziale Frage“ abzulösen. Damit war die Klassenfrage im emphatischen Sinne als politisch-praktische Frage eigentlich kaum noch eine zentrale PolitikOption für die Beteiligten. Letztlich verlief all das nicht gerade im Sande, aber eine maßgebliche Veränderung der gegebenen Herrschaftsverhältnisse konnte nicht erreicht werden. Das wurde nicht zuletzt deutlich am begrenzten Einfluss auf die Praxis der Gewerkschaften, die zeitweise manchen Bestrebungen der Studentenbewegung nahe gestanden hatten und einigen ihrer Protagonisten sogar einen gewissen Zugang zu ihrer Bildungsarbeit ermöglicht hatten. Exemplarisch hierfür kann Oskar Negts Soziologische Phantasie und exemplarisches Lernen (1968) stehen, das z.B. in der IG-Metall breite Verwendung fand. Aber das änderte wenig an der grundsätzlichen Einbindung der Gewerkschaften in den herrschenden Klassenkompromiss.

Es ist bezeichnend, dass in den Augen vieler Beteiligter die Gewerkschaften als zentrale Organisationen der Arbeiterbewegung, und damit diese selbst, zu einer „alten“ sozialen Bewegung mutierten, die letztendlich keine politischen Perspektiven zu bieten schien. Damit wurde die so verstandene „alte soziale Frage“, 
das im Kapitalverhältnis gründende, aber nicht in ihm aufgehende Klassenverhältnis nachrangig; zumal die anfangs gesuchte Arbeiterklasse als eine kämpfende nicht gefunden worden war. Stattdessen war man/frau auf Arbeiterinnen und Arbeiter getroffen, die zumindest in der BRD mehrheitlich an den Früchten eines prosperierenden Kapitalismus partizipierten und dies nicht zuletzt durch die Gewerkschaften zu steigern und abzusichern suchten; das schloss Arbeitskämpfe und Streiks nicht aus, aber das Kapitalverhältnis als solches trat dabei nicht ins Blick- und Handlungsfeld (vgl. Birke 2007). Die gewünschte neue Dimension von Gesellschaftlichkeit konnte darin nicht gefunden werden. An die Stelle der „alten“ trat die „neue soziale Frage“ und mit ihr die Neuen Sozialen Bewegungen, die Ungleichheit zwischen den Geschlechtern, der ruinöse Umgang mit „der Natur", wie er sich in Kohlekraftwerken, aber insbesondere in Atomkraftwerken manifestierte; Tschernobyl war eine Mahnung und tat seine Wirkungen.

Kein Wunder, dass Ulrich Becks „Risikogesellschaft“ (1986) einer der wenigen soziologischen Bestseller dieser Zeit war, in dem „die Klassenfrage“ letztlich verabschiedet wurde. Die Grünen schienen die lange gesuchte und nun endlich gefundene neue Avantgarde des gesellschaftlichen Fortschritts zu sein, bis hin zur Regierungsbeteiligung und der Mitverantwortung für die klassenpolitisch sehr bedeutsame Hartz-IV-Gesetzgebung.

\section{Klassentheoretischer Stillstand und Gleiswechsel}

Es wundert nicht, dass Klassen als Thema sozialwissenschaftlicher Untersuchungen und Debatten oder gar öffentlicher Diskussionen dann für eine längere Zeit kein eigentliches Thema waren. Die Mainstream-Soziologie beschritt weiter und verstärkt die alten Wege, die man mit neuem Vokabular aufzufrischen suchte. Neue Ungleichheiten waren angesagt, man diskutierte über Individualisierung und Differenzierung, tagte, woraus dann die üblichen Tagungsbände resultierten, aber recht eigentlich war die Luft raus. An die Stelle von Klassen traten Milieus, deren Verschiebungen man in immer ausgeklügelteren Varianten nachgehen konnte. Um Aufmerksamkeit zu gewinnen, musste man das schon verdichten und aufmotzen, indem die herrschende Gesellschaft zur „Erlebnisgesellschaft“ (Schulze 1992) geadelt wurde, die in sich immer wieder wiederholenden Symposien und medialem Hype besprochen wurde.

Sicher gab es Versuche, dem kritisch zu begegnen und die Klassenfrage im Spiel zu halten. Aber da man sich politisch und theoriestrategisch in der Defensive befand, handelte es sich vorwiegend um Kritiken an den vorherrschenden Denkfaulheiten oder um Aufgabenstellungen, die es zu bearbeiten gelte, was dann allerdings aufgrund institutioneller Gegebenheiten kaum oder nicht realisiert 
werden konnte. ${ }^{2}$ Statt vieler Belege sei hier nur auf die zwei Bände Ungleichheiten. Protheorie sozialer Ungleichheit und kollektiven Handelns von Veit Bader und Albert Benschop (1989/1991) hingewiesen, in denen ein Großteil der vorhandenen Literatur gründlich gesichtet und in fundierte Aufgabenstellungen umgesetzt wurde. Aber die Hoffnung auf eine Wiederentdeckung der Klassen, so ein späterer Band von Bader u.a. (1998) war allzu optimistisch und voraussetzungsvoll. Denn zwar wurde hier der Versuch unternommen, in einer Zusammenführung von historischen Untersuchungen und soziologischen Analysen der aktuellen herrschenden Verhältnisse eine Art Quintessenz der auch wissenschaftlichen Erfahrungen seit der Studentenbewegung mit ihren unterschiedlichen politischen Verlaufsformen zu ziehen. Aber diese wurde weder in der Zunft noch in der (linken) Öffentlichkeit in relevantem Maße beachtet. Die Bemühungen, eine breitere Diskussion zu entfachen, waren begrenzt, in der Regel blieb es bei den üblichen Kreisen (etwa um die Zeitschrift für Marxistische Erneuerung oder den Sozialismus), die irgendwie qua Existenz und Tradition die Klassenfrage immer im Blick hatten. Hier wandte man sich zunehmend den Gesichtern des neuen Kapitalismus (Herkommer 1999) und debattierte Verdeckte und offene Strukturen sozialer Ungleichheit (Bischoff/ Herkommer/Hüning (2002). Aber mit dem Ende des sog. realen Sozialismus und spätestens mit den Auswirkungen der „vom Westen“ eher ungewollten deutschen Einigung war der Zug auch hier eigentlich schon länger abgefahren.

Es sei denn, dass man ohnehin ein anderes Gleis gewählt hätte. Die von Karl Heinz Roth angestoßene Diskussion um Die Wiederkehr der Proletarität (1994) wirft ein grelles Licht auf sichtbare Veränderungen der Klassenverhältnisse und (häufig) vermutete Auswirkungen auf das Herrschaftsgefüge in seiner internationalen Ausprägung. In der Konstatierung und empirischen Fundierung eines grundlegenden Bruchs mit dem Fordismus und seinen sozialstaatlichen Implikationen ist das belebend. Aber gleichzeitig bleiben grundlegende Fragen offen. Unter Rückgriff auf den mit dem Begriff der Globalisierung angesprochenen Zusammenhang geht Roth von einer einheitlichen Tendenz des Kapitals weltweit aus, vom „Aufkommen der neuen Klassengesellschaft seit den achtziger Jahren“ (Roth 1994: 13). Die Folge sei „tendenziell [sic!] ein neues Proletariat in einer kapitalistischen Welt. Die bisherigen Klassenunterschiede der ersten, zweiten und dritten Welt“würden ,zunehmend nivelliert, miteinander verflochten und aneinander angepaßt“ (ebd.: 18). Das ist letztlich abstrakt gesetzt und nur unzureichend empirisch gesättigt, denn die Unterschiede zwischen der Lage von Arbeiter_innen in unterschiedlichen Weltregionen nahmen nicht ab, sondern zu. Roth unterscheidet nicht genügend zwischen „dem Kapital“ und Einzelkapitalen,

2 Das gilt auch für meine Beiträge aus diesen Jahren, die in Thien (2010) gesammelt sind. 
er unterschätzt Veränderungen innerhalb des Gesamtarbeitskörpers und landet letztlich bei einer Art Verelendungstheorie, die ausschließlich auf den Zwangsgesetzlichkeiten kapitalistischer Akkumulation gründet. Gleichzeitig verstrickt er sich dadurch in einen grundlegenden Widerspruch, dass er entgegen seiner behaupteten Angleichungstendenz bei den Lohnarbeiter_innen die gewerkschaftlich organisierten Kernschichten politisch abschreibt und das Potential für eine Veränderung nur noch in „Randgruppen“ sieht: Arbeitslosenbewegungen, Jobbergruppen, linksgewerkschaftliche Betriebsgruppen, scheinselbständige Arbeiter_innen etc., also dem, was heute als Prekariat diskutiert wird. Wie aber sollte daraus dann eine handlungsfähige Klasse werden, und das auch noch weltweit? Im Unterschied zu den althergebrachten Gewerkschaften mit ihren überholten Vorstellungen scheint die andere Arbeiterbewegung weiter zu wirken oder neu zu entstehen; aber wie wird sie zu einer Arbeiter_innenbewegung?

Zur Kenntnis genommen wurde auch das nur am Rande. Wer sollten die Adressat_innen sein? Wohl kaum Arbeiter_innen, die sich im Fordismus und auch darüberhinaus einigermaßen hatten einrichten können und denen es gelungen war, eine gewisse Planungssicherheit für ihr Leben zu erreichen. Blieben autonome Gruppierungen, die es durchaus gab, deren Einfluss auf politische Diskussionen aber gering geworden war. Versuche, eine breitere Diskussion zu eröffnen, scheiterten.

Eine zu beachtende und in gewissem Sinne symptomatische Ausnahme hiervon allerdings gab es, nämlich die Arbeiten von und die Diskussion um Pierre Bourdieu. Dieser hatte völlig zu recht auf den Unterschied zwischen „Klassen auf dem Papier" und realen Klassen hingewiesen und somit eine Fortführung des Klassendiskurses signalisiert. Gleichzeitig hatte er vor allem mit Die feinen Unterschiede (Bourdieu 1982) eine umfassende empirische Fundierung seiner Überlegungen vorgelegt, um schließlich aber keineswegs als Empirist zu erscheinen, sondern durch seine weiteren Publikationen und nicht zuletzt die zahlreichen Medienauftritte in die Garde der Großen Theoretiker eingereiht zu werden. Und als solcher wurde er dann auch hierzulande primär gehandelt (vgl. z.B. die Beiträge in Eder 1989 und Rademacher/Wiechens 2001). Langwierige Debatten um den Stellenwert zentraler bourdieuscher Begriffe, um „Habitus, ökonomisches und kulturelles Kapital“, oder um den ethnologischen bias seiner Theorie ließen die Klassenfrage jedoch in den Hintergrund geraten. Und Bourdieu trug letztlich dazu bei. Denn einerseits legte er immer neue Variationen seines Theoriekonzepts vor. Andererseits näherte er sich mit Fallstudien wie Das Elend der Welt (1993/1997) der subjektiven Verfasstheit von Arbeiter_innen; aber auch wenn die Ebene des alltäglichen Klassenkampfs (Bourdieu 1992: 148) immer präsent blieb, unterblieb doch eine klassentheoretische Verortung. Das gilt ebenso für seine Analyse „männlicher Herrschaft“ (Bourdieu 2012; Bock/Dölling/Krais 2007). 
Robert Castel hat dies dann in seiner weit ausholenden Studie Die Metamorphosen der sozialen Frage (1995/2000) übernommen. Ausgehend von der Problematik der Durchsetzung der kapitalistischen Gesellschaft führt er die unterschiedlichen Phasen der Transformation der Lohnarbeit - er nennt es "Metamorphosen“ - anschaulich vor Augen; dabei zeigt er detailliert, wie unterschiedliche Fassungen des „Wohlfahrtsstaates“ die zeitweise verheerenden Folgen der Lohnarbeit einzudämmen und in geordnete Bahnen zu lenken suchen. Das gilt insbesondere für die Zeit nach dem Zweiten Weltkrieg und die Phase des Fordismus, in der der Klassengegensatz in einem Klassenkompromiss stillgestellt schien und „Arbeit wie Kapital“ gleichermaßen am gesellschaftlichen Fortschritt zu partizipieren schienen. Allerdings blieb das, so immer noch Castel, Episode; denn die unaufhaltbar scheinende Verbesserung der ökonomischen Lage von Arbeitern in den 1960er Jahren verflachte zusehends ebenso wie der Ausbau ihrer staatlichen Zusatzabsicherung. In „der“ Lohnarbeit machten sich dann in den 1980er und -90er Jahren immer stärker neue interne Differenzierungen geltend.

Michael Vester et al. (1993/2001) haben im Anschluss an Bourdieu eine groß angelegte empirische Aufschlüsselung des sozialstrukturellen und habituellen Feldes der BRD vorgelegt und damit den „Wandel der Klassengesellschaft“ zu belegen versucht. Im Ergebnis unterscheiden sie in ihrer Typologie drei Stufen: die hegemonialen Milieus, die respektablen Volks- und Arbeitnehmermilieus und die unterprivilegierten Volks- und Arbeitnehmermilieus (504). Das ist, auch und gerade in der Veranschaulichung durch verschiedene Tableaus, einleuchtend und macht deutlich, dass die Vorstellung eines sich auch in den Personen ausdrückenden diametralen Gegensatzes von Lohnarbeit und Kapital, aber auch die einer völligen Vereinnahmung durch „das System“ gegenüber einer grundsätzlichen Ablehnung eben dieses Systems allzu vereinfachend ist. Gefragt sind die Zwischenräume und Übergänge, gefragt ist die interne Festigkeit der Milieus, die den Veränderungen der Kapitalakkumulation ebenso ausgesetzt sind wie politischen Einflussnahmen und neuen politischen Akzentsetzungen. Milieus sind relativ stabile Figurationen, was Änderungen nicht ausschließt, die aber nicht unmittelbar sichtbar sind. Das gilt etwa für die in langen Jahren vor und nach 1945 erfolgte Bindung von Arbeiter_innen an die SPD als Arbeiterpartei und ihre Reaktionen auf die neoliberale Hartz IV-Gesetzgebung durch SPD und Grüne oder die „Rente mit 67 “.

\section{Klassen in der Krise}

Wenn 40 Jahre nach Adornos Vortrag auf dem 1968er Soziologentag der Präsident der Deutschen Gesellschaft für Soziologie, Karl-Sigbert Rehberg, sich 2008 bei 
der Eröffnung des 34. Kongresses der Deutschen Gesellschaft für Soziologie gehalten sah, die soziale Frage und sogar die Klassenfrage wieder auf die offizielle Agenda soziologischer Thematiken zu setzen, dann musste etwas im Anzug oder passiert sein, dass selbst Soziolog_innen ums Nachdenken nicht herum kamen. Und dann kam zwischen 2007/2008 der harte Einschnitt, die Finanzmarktkrise, die manche als „Große Krise“, andere als „Vielfachkrise“ (Demirović u.a. 2011) oder als letzten Baustein eines „Epochenbruch" sahen, der den Postfordismus oder Neoliberalismus über diesen hinaus transformierte. Vieles schien im gravierenden Umbruch, was sowohl eine interne Neuzusammensetzung der Bourgeoisie(n) wie auch der Arbeiterklasse(n) beinhaltete, damit verbunden aber insbesondere Verschiebungen in und zwischen den großen Blöcken (USA, Europa, Asien, Lateinamerika, Russland) meint.

Soviel sollte klar sein: Eine Krise derartigen Ausmaßes, die den Weltmarkt zeitweise an den Rand eines Zusammenbruchs zu bringen schien und nur durch immense Eingriffe der Einzelstaaten und Staatenverbünde einigermaßen aufgefangen werden konnte, lud auch innerhalb der Linken geradezu zu Rückgriffen auf bekannte Argumentationsmuster ein, wie sie zeitweise in der Verelendungstheorie und dem tendenziellen Fall der Profitrate als allgemeine Krise des Kapitals verwendet wurden. Der Zusammenbruch schien manchen nahe, man sah sich bestätigt, behauptete eine allgemeine Verschlechterung der Lage von Lohnarbeiter_innen, wusste aber auch nicht recht damit etwas anzufangen.

Gegenüber solchen allzu luftigen Konstruktionen brachten andere die trotz aller historischen Modifikationen bestehende Grundkonstellation „des“ Kapitalismus zur Geltung. Man musste an die Kapitalform erinnern und einen erneuten Anstoß zur Lektüre des Kapitals geben. Klassentheoretisch schlug sich das, ähnlich wie schon bei der 68er Student_innen-Bewegung nieder in einer insbesondere studentischen Kapital-Lese-Bewegung, die immer nützlich ist, deren Auswirkungen für eine Analyse der Klassenverhältnisse aber begrenzt bleiben (vgl. z.B. Ellmers 2007).

Manche Poststrukturalisten sahen sich bestätigt und berauschten sich geradezu an den Manifestationen einer neu gesehenen Widerständigkeit allüberall. Seien es die Blockupy-Aktionen oder der Arabische Frühling, die Abwehrkämpfe der griechischen, spanischen oder portugiesischen Bevölkerung gegen eine Subordinierung durch die dominanten Gruppen/Länder der EU, alles geriet zum Aufstand für eine verheißungsvolle Zukunft. Endlich schien gegenüber den Verkarstungen eines dem Kapital unterworfenen Prozesses „der subjektive Faktor der Geschichte“ (Seibert 2012) zurück und „den Humanismus“ zu verwirklichen. Diese Erwartungen wurden inzwischen durch die Entwicklung in den arabischen Ländern, die sich in ihrer gesellschaftlich-politischen Besonderheit als gar nicht verallgemeinerungsfähig erwiesen, enttäuscht. Auch die Proteste in den europäischen Ländern 
blieben angesichts der Peripherisierung von südlichen Ländern innerhalb der EU, insbesondere in Griechenland, Spanien und Portugal v.a. defensiv.

Ihre theoretische Weihe erhielt die Aufbruchshoffnung durch Michael Hardt und Antonio Negri, die mit ihren Publikationen in autonomen und globalisierungskritischen Kreisen und in der bürgerlichen Öffentlichkeit für Aufsehen sorgten. In der Konstatierung einer grundsätzlichen Veränderung des Kapitalismus hin zu einem informationellen Kapitalismus, dem alle Individuen subordiniert seien, lösten sie den Klassengegensatz letztlich auf, indem sie ihn plan verallgemeinerten und zum Gegensatz von Arm und Reich, von Herrschenden und Subordinierten ummodelten. In weit ausholender Manier eines Streifzuges durch die gesamte Philosophiegeschichte und viele Aufstandsbewegungen erschließen sie sich ihren Begriff der Multitude, der nichts anderes beinhalten soll als eine ,politische[n] Anthropologie des Widerstands“(Hardt/Negri 2010: 253):

„Die Partei der Armen hingegen beruht nicht auf der Exklusivität eines Teils der Gesellschaft, sondern sie ist eine Formation all jener, die in die Mechanismen der gesellschaftlichen Produktion eingespannt sind, ungeachtet ihres Ranges oder Besitzes, in all ihrer Verschiedenheit, und beseelt durch eine offene und plurale Produktion der Subjektivität. Durch ihre bloße Existenz stellt die Multitude der Armen eine objektive Gefahr für die Republik des Eigentums dar." (2010: 60).

Trotz aller Anleihen bei Marx und Gramsci, bei Althusser und Foucault und vielen anderen dominiert letztlich das aus der Geschichte marxistischen Denkens altbekannte Argument, dass das Kapital die Voraussetzungen zu seiner Abschaffung besorgen wird (310), nur dass nun der Zeitpunkt endgültig gekommen scheint: "Jetzt, endlich, steht die Revolution auf der Tagesordnung“ (351; zur Kritik vgl. z.B. Thien 2010; Tomba/Bellofiore 2010; Stricker/Tulatz 2014).

Auch wenn Karl-Heinz Roth und Marcel van der Linden einen ähnlichen Begriff verwenden, nämlich den des „Multiversums“, sind sie trotz aller Nähe zum Gestus der Überlegungen von Hardt und Negri ein gutes Stück näher an den empirischen Ausprägungen des Kapitalverhältnisses (siehe insbes. van der Linden 2008) und trotz aller Kritik auch an Marx. Wenn sie allerdings zusammenfassen, dass die unterschiedlichen Formen der Mehrwertabpressung des transnationalen Kapitals folgerichtig zusammenschießen in die „Weltarbeiterklasse“ und sich daher „nicht als homogenes Gebilde, sondern als ein Multiversum von Schichten und Gesellschaftsgruppen, dessen Lernprozesse, Erfahrungen, Zusammenschlüsse und Handlungen nicht so sehr durch die einzelnen Subjekte, sondern viel stärker von Familien, Gemeinden, Brüder- und Schwesternschaften, Hilfs- und Gewerkvereinen und Geheimbünden getragen werden“ (Roth/van der Linden 2009: 560), dann bleibt dies ein Wunsch - man könnte sagen, ein verständlicher Wunsch, den wir als Aufgabenstellung verstehen sollten (vgl. die Kritik von Kößler/Wienold 2013: 351ff). 
Selbstverständlich kritisieren sowohl Hardt/Negri wie auch Roth/van der Linden die Unterdrückung von Frauen und wissen sich einig mit maßgeblichen Strömungen des Feminismus. Unübersehbar ist, wie sehr insbesondere Frauen aus der früher sogenannten Dritten Welt in die Unterordnungsprozesse des transnationalen Kapitals einbezogen sind. Ein Problem scheint aber zu sein, dass der Feminismus in gewissem Sinne an seinem eigenen Erfolg litt. Denn zwar war es, zumindest hierzulande und v.a. im akademischen Feld, gelungen, den Konkurrenznachteil von Frauen gegenüber ihren männlichen Kollegen abzubauen, und dies etwa im gender mainstreaming auch auf andere Bereiche auszuweiten, aber gleichzeitig war eine Stagnation der feministischen als einer politisch relevanten Bewegung kaum zu übersehen. Das führte zu selbstkritischen Befragungen der eigenen Geschichte (vgl. etwa Kontos 2004, Fraser 2004, Acker 2010), in denen mit politischer Stoßrichtung an die 1970er-Fragen nach Klasse-Geschlecht angeknüpft wurde. So fordert Fraser eine „nicht-identitäre feministische Politik“ (2004: 465), in der sowohl die, vereinfacht gesagt, ökonomische Lage wie die patriarchale Unterordnung aufgehoben werden soll. Lars Kohlmorgen (2004: 261ff) versucht den Milieu-Begriff nutzbar zu machen, während Jörg Nowak (2009) bezugnehmend auf die gegebene Arbeitsteilung und ihre Modifikationen die Bedeutung von Maßnahmen der Familienpolitik für die interne Differenzierung von Männern und Frauen und auch von Fraktionen innerhalb der Arbeiter_innenklasse geltend macht (ebd.: 62ff und 245ff). Dabei bezieht er sich nicht zuletzt auf die Arbeiten von Frigga Haug (2001), die innerhalb der feministischen Bewegung immer wieder eine Rückbesinnung auf die Klassenproblematik einforderte.

Eine solche erfolgte nicht unmittelbar, aber inzwischen ist diese Problematik Teil einer hierzulande geradezu ausufernden Debatte um die Intersektionalitätvon „race, class und gender“ (vgl. z.B. Knapp/Wetterer 2003, Klinger/Knapp 2008, Smykalla/Vinz 2011, Hess/Langreiter/Timm 2011). Das ist zu umfangreich, um es hier mehr als anzusprechen. Eine Bemerkung aber sei gewagt: So weit ausholend wie detailliert die Debattenbeiträge auch sind, bisher scheinen mir Versuche einer theoretischen Grundsatzklärung (siehe vor allem die Beiträge von Cornelia Klinger $(2008,2014)$ und methodische Vorschläge (vgl. Winker/ Degele 2009) zu überwiegen, während materiale Analysen kaum zu finden sind.

\section{Der Debatte letzter Stand - und alle Fragen offen?}

Es ist geradezu erstaunlich, dass gegenüber den hochfliegenden Vorstellungen von einer Weltarbeiter_innenklasse oder gar einer unmittelbar bevorstehenden Revolution einer Multitude Gruppierungen, die gemeinhin eher als Vertreter der reinen 
Lehre gegolten hatten, sich einlassen auf wichtige empirische Veränderungen des zeitgenössischen Kapitalismus und deren offensichtlichen Auswirkungen auf das, was man Arbeiterklasse und Arbeiterbewegung nennt. Gemeint ist die Zeitschrift für Marxistische Erneuerung, die 2010 eine ziemlich offene Diskussionsrunde über Klassen eröffnete und sich dabei, wenn ich recht interpretiere, letztlich von den lange Zeit geltenden substanzhaften Vorstellungen von dem einen Proletariat, auf das manche immer gehofft hatten, verabschiedete.

Angestoßen durch Rezensionen neuerer Publikationen zur Klassentheorie (Thien 2010,2011), in denen deren Weiterführung und Aktualisierung gefordert wurde, intervenierte Lothar Peter mit einem kritischen Grundsatzeinspruch „Was machen wir mit dem Klassenbegriff?“ (2010), indem er wichtige Desiderata anführte und letztlich für eine Beendigung des bekannten Klassendiskurses plädiert. Seine Argumente sind nicht neu, aber ernsthaft, kenntnisreich und substanziell. In aller Kürze zusammengefasst vertritt Peter eine sozioökonomische Spielart des Klassenbegriffs, der aus einer Verbindung von ökonomischer Lage und Sozialcharakter besteht und sich in Handlungen geltend macht. Genau diese Bestandteile aber treten seiner Meinung nach im Laufe der Jahrzehnte nach dem Zweiten Weltkrieg hierzulande durch die Erosion von Kollektivität, Segmentierung und sozialen Aufstieg wie Abstieg (vgl. ebd.: 137) derart auseinander, dass es keinen Sinn mehr mache, von einer Arbeiterklasse zu sprechen.

Dieter Boris (2010) räumt die von Peter ins Feld geführten empirischen Veränderungen ein und betont deren Wichtigkeit, gleichwohl besteht er auf dem Klassenbegriff; denn dieser ,ist ja nicht nur oder ausschließlich ein empirischer Begriff, der vor allem Klassen in actu beschreibt oder abbildet, sondern er ist auch - und vielleicht sogar vor allem - ein analytisch-theoretischer und heuristischer Begriff, der die objektive 'Spaltung', 'Verelendung' und den 'Antagonismus' in den Herrschaftsverhältnissen - ...verständlich und besser analysierbar machen soll.“ (ebd.: 148) Es komme eben darauf an, die spezifische Eingebundenheit von Individuen in den kapitalistischen Produktions- und Reproduktionsprozess und unterschiedliche Handlungsmöglichkeiten zur Kenntnis zu nehmen. Das gelte nicht zuletzt für die herrschende Klasse. Gerade die von Peter zu recht ins Feld geführte „Auffächerung und Segmentierung der subordinierten und subalternen Klassengruppierungen“ (ebd.: 150) stellten eine Herausforderung für die Klassentheorie dar und belegten zugleich ihre Notwendigkeit.

In einem weiteren Beitrag zur Debatte plädieren Klaus Dörre und Steffen Liebig (2011) ausdrücklich für eine Beibehaltung des Klassenbegriffs, den sie mit dem Ausbeutungs- sprich Kapitalverhältnis grundgelegt sehen. Dieses Plädoyer - zwischenzeitlich hat der unermüdliche Klaus Dörre (teilweise mit CoAutor_innen:vgl. Dörre/Castel 2009, Dörre u.a. 2013; Dörre/Happ/Matuschek 2013; Haipeter/Dörre 2011) weitere Ergebnisse seiner Beschäftigung mit der 
Klassenproblematik vorgelegt - soll illustrativ für den Zwischenstand der lang anhaltenden klassentheoretischen Diskussion herangezogen werden.

Unter Rückbezug sowohl auf den methodischen Status von Marx' Kapital wie auch seiner historischen Schriften verweisen die Autoren auf die Grundkonstellation „beherrschter Klassen“, womit richtigerweise die Arbeitskraft-Besitzer gemeint sind, die sich „Konkurrenz, Spaltung und soziale(r) Fragmentierung“ (Dörre/ Liebig 2011: 29) ausgesetzt sehen; eine eindeutige Richtung sozialer Kämpfe, womöglich noch als lineare Prozesse, ergebe sich daraus nicht. So weit, so gut. Schwierig wird es dann, wenn Dörre/Liebig auf die aktuellen Prozesse zu sprechen kommen und diese zu erklären versuchen. Bestimmend dabei sind die Begriffe kapitalistische Landnahme, den Klaus Dörre in verschiedenen Zusammenhängen benutzt hat (Dörre 2011, 2010a) und sekundäre Ausbeutung (30ff). - Was ist gemeint? Auch hier muss ich mich auf wenige Anmerkungen beschränken.

Die Autoren suchen nach einem Prinzip für all das, was vor unseren Augen abläuft, und sind dabei auf Rosa Luxemburg und Burkart Lutz gestoßen, deren Landnahme-Theorem besagt, dass der Kapitalismus sich nicht auf seinen eigenen Grundlage zu reproduzieren vermag und deshalb beständig auf die Okkupation eines nichtkapitalistischen Anderen angewiesen ist. Aber ist das tatsächlich so? Ist das nicht viel zu allgemein? Ordnet sich das Kapital tatsächlich alle nichtkapitalistischen Verhältnisse unter - welche gibt es noch und wo? - oder sollte man nicht eher sagen, dass es sich nach innen und außen richtet und immer erneute Anläufe zu seiner Ausweitung unternimmt, aber dabei auch auf Grenzen stößt, die erst zu überwinden sind, was keineswegs immer gelingt? Ja, auch der Zwang zur Mehrwertproduktion kann auf Grenzen stoßen, die nicht einfach überwunden werden können. Auch aus einer Krise ergeben sich keine eindeutigen Handlungsfolgen; hier wäre der von Dörre zugrunde gelegte Krisenbegriff, der weitgehend normativ aufgeladen ist (vgl. Röttger 2011: 649) genauer zu bestimmen. Statt diese möglichen Grenzen, die historisch-politischer Art sind, auszuloten, verlängern Dörre/Liebig das Landnahme-Theorem zu dem einer sekundären Ausbeutung, das auch noch in vorkapitalistischen Verhältnissen wurzeln soll. Das „Perpetuum mobile kapitalistischer Landnahmen“" (Dörre/Liebig 2011:31) funktioniere und mache sich etwa in der Benachteiligung von Frauen geltend. Eine solche Benachteiligung ist ziemlich offensichtlich, aber es enthebt nicht von ihrer genauen Entschlüsselung. Die interne Differenzierung der Arbeiter_innenklasse wäre erst noch konkret aufzuweisen. Die Autoren tun sich m. E. keinen Gefallen, wenn sie unterscheiden zwischen "primärer" und "sekundärer Ausbeutung“, die sie dann auch noch „formationsunspezifisch“ aufladen (ebd.), was ja wohl heißen soll, dass sie nicht „dem Kapitalismus“, den es in Reinform nie gegeben hat, geschuldet seien. Aber macht das nach über 200 Jahren Kapitalismus noch Sinn, wenn es je welchen gemacht hat? 
Überzogen sind auch die Ausführungen zu den klassenmäßigen Auswirkungen des Finanzmarktkapitalismus. Zwar führen Dörre/Liebig anschaulich vor Augen, in welchem Ausmaß hier Verschiebungen in den Ausbeutungsverhältnissen und den Umverteilungen stattfinden, aber sie lassen sich in gewissem Sinne von den Daten blenden, die auf ein Immer-weiter-So hinweisen, während wir doch seit einiger Zeit eine Neuzusammensetzung der Klasse der Lohnarbeiter_innen und möglicherweise eine Kräfteverschiebung innerhalb der herrschenden Klassen erleben, die unbedingt zu beachten wäre.

Wichtig sind ihre Ausführungen zur internen Differenzierung der „subalternen Klassen“ (ebd.: 36), der Lohnabhängigen also, wie man schlichter sagen könnte. Drei Klassenfraktionen werden unterschieden: a) Hochqualifizierte Beschäftigte, b) qualifizierte Angestellte und Arbeiter in kommandierter Beschäftigung und c) Beschäftigte im prekären Sektor als „,sozial neu zusammengesetzte Unterklasse (resp. Unterklassen)“ (ebd.: 37). Aber was bedeutet die Schlussfolgerung, dass „... Arbeiterklasse auch im nationalen Rahmen entwickelter Kapitalismen im Plural definiert werden [muss]“ (ebd.)? Gibt es in Deutschland drei Arbeiter_innenklassen? Tatsächlich wollen es Dörre und Liebig so verstanden wissen. Sicherlich können sie sich dabei auf den unterschiedlichen Zugang der betreffenden Gruppen zu Machtressourcen und, wenn man es hoch hängt, auf einen unterschiedlichen Habitus beziehen; aber macht es gleichwohl nicht eher Sinn von drei Fraktionen von Lohnabhängigen zu sprechen, deren Mitglieder sich voneinander unterscheiden, denen aber gemeinsam ist, dass sie sich nicht als solche einer Klasse verstehen? Macht es Sinn, von den Stammarbeitern als „Beherrschte in herrschender Stellung“ (ebd.: 39) zu sprechen? Was meint dabei Herrschaft? Und sollten hierbei nicht die internen Differenzierungen innerhalb der Gruppierungen nach Geschlecht und Ethnie inhaltlich und empirisch aufgenommen werden, wie sie sich etwa in der Intersektionalitätsdebatte ausdrücken, statt sie nur am Rande zu erwähnen?

Klaus Dörre und Steffen Liebig sprechen ähnliche Fragen in ihrem Forschungsprogramm zwar an, aber letztlich bleiben sie offen. Ihre abschließenden Hinweise zu unterschiedlichen Formen der sozialen Konfliktaustragung, die sich als Vielfalt verschiedener Varianten darstellt, beginnend mit „Labor Unrest“, „verrückten Aufständen“ etwa in Großstadtvorstädten bis hin zu Volksaufständen (ebd.: 40f) nehmen bekannte Motive aus der Geschichte der Arbeiter_innen- und Protestbewegung auf. Sie belegen erneut, dass der Klassenantagonismus eben nicht eindeutige Implikationen für Klassenauseinandersetzungen enthält; diese selbst sind in ihrer konkreten Ausprägung zu entschlüsseln (vgl. als Versuch Thien 2013). 


\section{Statt eines Resümees}

Ich versuche gar nicht erst, eine Art Quintessenz der vorherigen Ausführungen zu ziehen, sondern verweise auf drei bedenkenswerte Auffassungen zur Klassenthematik aus einem lesenswerten Buch mit dem allerdings schrecklichen Titel Hello Marx (Bescherer/Schierhorn 2009). Dort hält Alex Demirović fest: „Die Existenz von Klassen, so könnte man sagen, ist gar nichts anderes als die Art ihres Kampfes“. (2009: 80). Das ist so, wie man seit E.P. Thompson weiß, aber was ist, wenn „die Klassen“ nicht kämpfen, gibt es sie dann nicht? Bernd Röttger sieht "Suchstrategien einer sich neu politisch konstituierenden subalternen Klasse“ (2009: 95); aber wie verlaufen sie und werden sie auch erfolgreich sein? Manuela Bojadžijev (2009: 143) hebt demgegenüber auf neue Formen der Subjektivität ab und warnt vor der Suche nach einem privilegierten Subjekt einer Veränderung, aber wie könnten sich unterschiedliche Subjekte verbinden?

\section{Literatur}

Acker, Joan (2010): Geschlechterfrage, Kapitalismus und Globalisierung, in: Beck, Ulrich/Poferl, Angelika (2010): 542-578.

Adorno, Theodor W. (1968/1980): Anmerkungen zum sozialen Konflikt heute, in: Gesammelte Schriften Bd. 8, Frankfurt/M.: Suhrkamp:177-194.

- (1942/): Reflexionen zur Klassentheorie, in: Gesammelte Schriften, Bd. 8, Frankfurt/M. : Suhrkamp: 373-391.

- (1993): Einleitung in die Soziologie, Nachgelassene Schriften Bd. 15: Frankfurt/M.: Suhrkamp. Althusser, Louis (1977): Ideologie und ideologische Staatsapparate, Hamburg/Westberlin: VSA.

Apeldoorn, Bastiaan van (2013): The European capitalist class and the crisis of its hegemonic project, in: Panitch, Leo/Albo, Greg/Chibber, Vivek (ed.): Socialist Register 2014: Registering Class, London: Merlin et. al., Hamburg: 189-206.

Aulenbacher, Brigitte/Riegraf, Birgit (2013): Kapitalismus und Krise - eine Frage von Ökonomie und Klasse? in: Atzmüller u.a. (Hrsg.): Fit für die Krise? Perspektiven der Regulationstheorie, Münster: Westfälisches Dampfboot: 90-110.

Arps, Jan Ole (2011): Frühschicht. Linke Fabrikintervention in den 70er Jahren, Berlin/Hamburg: Assoziation A.

Bader, Veit M./Benschop, Albert (1989/1991): Ungleichheiten. Protheorie sozialer Ungleichheit und kollektiven Handelns, 2 Bde., Opladen: Leske + Budrich.

Bader, Veit M./Benschop, Albert/Krätke, Michael R./Treeck, Werner van (Hrsg.)(1998): Die Wiederentdeckung der Klassen, Hamburg: Argument.

Balibar, Etienne/Wallerstein, Immanuel (1990): Rasse, Klasse, Nation, Hamburg: Argument.

Beauvoir, Simone (1968): Das andere Geschlecht, Reinbek: Rowohlt.

Beck, Ulrich (1986): Die Risikogesellschaft, Frankfurt/M.: Suhrkamp.

Beck, Ulrich/Poferl (Hrsg.)(2010): Große Armut, großer Reichtum. Zur Transnationalisierung sozialer Ungleichheit, Frankfurt/M.: Suhrkamp.

Beckenbach, Niels (1973): Klassenlage und Bewußtseinsbildung der technisch-wissenschaftlichen Lohnarbeiter. Zur Diskussion über die „Technische Intelligenz“, Frankfurt/M. 
Becker-Schmidt, Regina/Knapp, Gudrun Axeli (1985): Arbeiterkinder gestern - Arbeiterkinder heute. Erziehungsansprüche und-probleme von Arbeiterinnen im intergenerativen Vergleich, Bonn: Verlag Neue Gesellschaft.

Becker-Schmidt, Regina (2007): „Class“, „gender“, „ethnicity“, „race“: Logiken der Differenzsetzung, Verschränkungen von Ungleichheitslagen und gesellschaftliche Strukturierung, in: Klinger, Cornelia/Knapp, Gudrun-Axeli/Sauer, Birgit (2007): 56-83.

Beer, Ursula (Hrsg.)(1987): Klasse Geschlecht. Feministische Gesellschaftsanalyse und Wissenschaftskritik, Bielefeld: AJZ.

Beerhorst, Joachim/Demirović, Alex/Guggemos, Michael (Hrsg.) (2004): Kritische Theorie im gesellschaftlichen Strukturwandel, Frankfurt/M.: Suhrkamp.

Bergmann, Joachim/Brandt, Gerhard/Körber, Klaus/Mohl, Hans-Theodor/Offe, Claus (1969): Herrschaft, Klassenverhältnisse und Schichtung, in: Adorno, T.W. (Hrsg.): Spätkapitalismus oder Industriegesellschaft? Stuttgart: 67-87.

Bescherer, Peter/Schierhorn, Karen (Hrsg.)(2009): Hello Marx. Zwischen „Arbeiterfrage“ und sozialer Bewegung heute, Hamburg: VSA.

Bescherer, Peter (2013): Vom Lumpenproletariat zur Unterschicht, Frankfurt/M.: Campus.

Birke, Peter (2007): Wilde Streiks im Wirtschaftswunder, Frankfurt/M.: Campus.

Bischoff, Joachim/Herkommer, Sebastian/Hüning, Hasko (2002): Unsere Klassengesellschaft, Hamburg: VSA.

Bock, Ulla/Dölling, Irene/Krais, Beate (Hrsg.)(2007): Prekäre Transformationen. Pierre Bourdieus Soziologie der Praxis und ihre Herausforderung für die Frauen-und Geschlechterforschung (Querelles. Jahrbuch für Frauen- und Geschlechterforschung Bd. 12), Göttingen: Wallstein.

Bojadžijev, Manuela (2009): Rassismus und Migration in der marxistischen Klassenanalyse, in: Bescherer, Peter/Schierhorn, Karen (2009): 132-144.

- (2012): Die windige Internationale, Münster: Westf. Dampfboot.

Boltanski, Luc (1982/1990): Die Führungskräfte. Die Entstehung einer sozialen Gruppe, Frankfurt/M.: Campus.

Boris, Dieter (2010): Was machen wir mit dem Klassenbegriff? Replik auf Lothar Peter, in: $Z$. Zeitschrift für marxistische Erneuerung, 21. Jg., H. 82: 146-150.

Bourdieu, Pierre (1982): Die feinen Unterschiede, Frankfurt/M.: Suhrkamp.

- (1985): Sozialer Raum und „Klassen“, Frankfurt/M.: Suhrkamp.

- (1987/1992): Rede und Antwort, Frankfurt/M.: Suhrkamp.

- (1998/2012): Die männliche Herrschaft, Frankfurt/M.: Suhrkamp.

Bourdieu, Pierre et al. (1993/1997): Das Elend der Welt, Konstanz: UVK.

Brandt, Gerhard (1990): Arbeit, Technik und gesellschaftliche Entwicklung. Transformationsprozesse des modernen Kapitalismus 1971-1987, Frankfurt/M.: Suhrkamp.

Brede, Carola (2012): Angestellte - ein unverstandenes Lohnarbeitsverhältnis, Frankfurt/M.: Brandes \& Apsel.

Castel, Robert (2000): Die Metamorphosen der sozialen Frage, Konstanz: UVK.

Demirović, Alex (2004): Kritische Gesellschaftstheorie und Gesellschaft, in: ders. (Hrsg.): Modelle kritischer Gesellschaftstheorie, Stuttgart: Metzler, 1-27.

- (2009): Staatliche Herrschaft und die politische Konstruktion von Klassen, in: Bescherer, Peter/ Schierhorn, Karen (2009): 62-88.

- (2011): Kritische Gesellschaftstheorie und die Vielfalt der Emanzipationsperspektiven, in: PROKLA, 41. Jg., H. 165: 519-543.

Demirović, Alex/Dück, Julia/Becker, Florian/Bader, Pauline (Hrsg.)(2011): VielfachKrise im finanzdominierten Kapitalismus, Hamburg: VSA.

Deppe, Frank (2013): Probleme der politisch-strategischen Positionierung der Linken, in: Das Argument. H. 301: 167-180. 
Dörre, Klaus (2010), Landnahme und soziale Klassen. Zur Relevanz sozialer Klassen, in: Thien (2011): 113-151.

- (2010a): Soziale Klassen im Prozess kapitalistischer Landnahmen, in: Bude, Heinz/Damitz Ralf M./Koch, André (Hrsg.): Marx. Ein toter Hund, Hamburg: 198-236.

Dörre, Klaus/Behr, Michael/Eversberg, Dennis/Schierhorn, Karen (2009): Krise ohne Krisenbewusstsein? Zur subjektiven Dimension kapitalistischer Landnahmen, in: PROKLA, 39. Jg., H. 157: 559-576.

Dörre, Klaus/Castel, Robert (Hrsg.) (2009): Prekarität, Abstieg, Ausgrenzung. Die soziale Frage am Beginn des 21. Jahrhunderts, Frankfurt/M.: Campus.

Dörre, Klaus/Liebig, Steffen (2011): Sekundäre Ausbeutung und Prekarität - Herausforderungen für die Klassentheorie, in: Z. Zeitschrift für marxistische Erneuerung, 22. Jg., H. 87: 25-43.

Dörre, Klaus u.a. (2013): Bewährungsproben für die Unterschicht? Soziale Folgen aktivierender Arbeitsmarktpolitik, Frankfurt/New York: Campus.

Dörre, Klaus/Happ, Anja/Matuschek, Ingo (Hrsg.) (2013): Das Gesellschaftsbild der LohnarbeiterInnen, Hamburg: VSA.

Eder, Klaus (1989): Klassenlage, Lebensstil und kulturelle Praxis. Beiträge zur Auseinandersetzung mit Pierre Bourdieus Klassentheorie, Frankfurt/M.: Suhrkamp.

Ellmers, Sven (2007): Die formanalytische Klassentheorie von Karl Marx, Duisburg: Universitätsverlag Rhein-Ruhr.

Fraser, Nancy (2004): Feministische Politik im Zeitalter der Anerkennung, in: Beerhorst, Joachim/ Demirovic, Alex/Guggemos (2004): 453-474.

Friese, Marianne (1991): Frauenarbeit und soziale Reproduktion. Eine Strukturuntersuchung zur Herausbildung des weiblichen Proletariats im Übergangsprozeßzur bürgerlich-kapitalistischen Gesellschaft - dargestellt an der Region Bremen, Bremen: Forschungsreihe des Forschungsschwerpunkts „Arbeit und Bildung“, Bd. 20.

Grohmann, Peter/Sackstetter, Horst (Hrsg.) (1979): plakat. 10 Jahre Betriebsarbeit bei DaimlerBenz, Berlin: Rotbuch.

Haipeter, Thomas/Dörre, Klaus (Hrsg.)(2011): Gewerkschaftliche Modernisierung, Wiesbaden: VS. Hardt, Michael/Negri, Toni (2002): Empire, Frankfurt/M.: Campus.

- (2004): Multitude, Frankfurt/M.: Campus.

- (2010): Common Wealth, Frankfurt/M.: Campus.

Haug, Frigga (2001): Zur Theorie der Geschlechterverhältnisse, in: Das Argument, 43.Jg., Nr. 243: 761-787.

Herkommer, Sebastian (Hrsg.)(1999): Soziale Ausgrenzungen. Gesichter des neuen Kapitalismus, Hamburg: VSA.

Hess, Sabine/Langreiter, Nicola/Timm, Elisabeth (2011): Intersektionalität revisited, Bielefeld: transcript.

Hirsch, Joachim (1995): Dernationale Wettbewerbstaat, Berlin/Amsterdam: Edition ID-Archiv. Hirsch, Joachim/Roth, Roland (1986): Das neue Gesicht des Kapitalismus, Hamburg: VSA.

Hirsch, Joachim/Wissel, Jens (2011): Transnationalisierung der Klassenverhältnisse, in: Thien (2011): 287-309.

Hoss, Willi (2006): „Komm ins Offene, Freund. “Autobiographie, Münster: Westf. Dampfboot.

Huws, Ursula (2013): The underpinnings of class in the digital age: living, labour and value, in: Panitch, Leo/Albo, Greg/Chibber, Vivek (ed.): Socialist Register 2014: Registering Class, London: Merlin et. al.: 80-107.

Institut für Marxistische Studien und Forschungen (1972/1973/1974): Klassen-und Sozialstruktur $\operatorname{der}$ BRD 1950-1970, in 3 Bänden, Frankfurt/M.

Kadritzke, Ulf (1975): Angestellte - die geduldigen Arbeiter, Frankfurt/M.: Europäische Verlagsanstalt. 
- (1982): Angestellte als Lohnarbeiter. Kritischer Nachruf auf die deutsche Kragenlinie, in: Schmidt, Gert u.a. (Hrsg.): Materialien zur Industriesoziologie (Sonderheft 24 der Kölner Zeitschrift für Soziologie und Sozialpsychologie),.

Klinger, Cornelia/Knapp, Gudrun-Axeli/Sauer, Birgit (Hrsg.) (2007): Achsen der Ungleichheit. Zum Verhältnis von Klasse, Geschlecht und Ethnizität, Frankfurt/M.: Campus.

Klinger, Cornelia/Knapp, Gudrun-Axeli (Hrsg.) (2008): Überkreuzungen. Fremdheit, Ungleichheit, Differenz (Forum Frauen- und Geschlechterverhältnisse Bd. 23), Münster: Westf. Dampfboot.

Klinger, Cornelia (2008): Überkreuzende Identitäten - Ineinandergreifende Strukturen. Plädoyer für einen Kurwechsel in der Intersektionalitätsdebatte, in: Klinger/Knapp, a.a.O.: 38-67.

- (2014): Für einen Kurswechsel in der Intersektionalitätsdebatte, auf: Portal Intersektionalität, abgerufen am 25.03.2014.

Koch, Max (2006): Poulantzas' Beitrag zur Klassen- und Sozialstrukturanalyse, in: Bretthauer, Lars/Gallas, Alexander/Kannankulam, John/Stützle, Ingo (Hrsg.): Poulantzaslesen, Hamburg: VSA: 120-136.

Kohlmorgen, Lars (2004): Regulation, Klasse, Geschlecht. Die Konstituierung der Sozialstruktur im Fordismus und Postfordismus, Münster: Westf. Dampfboot.

Kontos, Silvia (2004), Brüche - Aufbrüche - Einbrüche, in: Beerhorst/Demirović/Guggemos (2004): 427-452.

Kößler, Reinhart/Wienold, Hanns (2013): Gesellschaft bei Marx, 2. korr. u. erw. Ausgabe Münster: Westf. Dampfboot.

Kracauer, Siegfried (1929/1971): Die Angestellten, Frankfurt/M.: Suhrkamp.

Krahl, Hans-Jürgen (1971): Konstitution und Klassenkampf. Zur historischen Dialektik von bürgerlicher Emanzipation und proletarischer Revolution, Frankfurt/M.: Neue Kritik.

Kronauer, Martin (2002): Exklusion, Frankfurt/M.: Campus.

Kudera, Werner/Mangold, Werner/Ruff, Konrad/Schmidt, Rudi/Wenzke, Theodor (1979): Gesellschaftliches und politisches Bewußtsein von Arbeitern, Frankfurt/M.: EVA.

Laclau, Ernesto/Mouffe, Chantal (1991): Ideologie undradikale Demokratie. Zur Dekonstruktion des Marxismus, Wien: Passagen.

Lucas, Erhard (1970): Märzrevolution im Rubrgebiet, Frankfurt/M.: März Verlag.

- (1976): Zwei Formen von Radikalismus in der deutschen Arbeiterbewegung, Frankfurt/M.:Roter Stern.

- (1983): Das Scheitern der deutschen Arbeiterbewegung, Basel/Frankfurt/M.:Stroemfeld/Roter Stern.

Lukács, Georg (1922/1967): Geschichte und Klassenbewußtsein, Amsterdam: Thomas de Munter.

Lüdtke, Alf (1993): Eigensinn. Fabrikalltag, Arbeitererfahrungen und Politik vom Kaiserreich bis in den Faschismus, Hamburg: Ergebnisse [Neuauflage in Vorbereitung].

Mason, Timothy W. (1982): Die Bändigung der Arbeiterklasse im nationalsozialistischen Deutschland, in: Sachse, Carola/Siegel, Tilla/Spode, Hasso/Spohn, Wolfgang: Angst, Belohnung, Zucht und Ordnung, Opladen: Westdeutscher Verlag, 11-53.

Marx, Karl (1969): Das Kapital, Bd. 3, MEW Bd. 25, Berlin: Dietz.

- (1974): Grundrisse der Kritik der politischen Ökonomie, Berlin: Dietz.

- (1969): Resultate des unmittelbaren Produktionsprozesses, Frankfurt/M.: Neue Kritik.

Mauke, Michael (1970): Die Klassentheorie von Marx und Engels, Frankfurt/M.: Europäische Verlagsanstalt.

Meschkat, Klaus/Negt, Oskar (Hrsg.) (1973): Gesellschaftsstrukturen, Frankfurt/M.: Suhrkamp. Meyer, Ahlrich (1999): Die Logik der Revolten, Berlin/Hamburg: Assoziation A.

Milhofer, Petra (1973): Familie und Klasse, Frankfurt/M.: Fischer.

Negt, Oskar (1968): Soziologische Phantasie und exemplarisches Lernen, Frankfurt/M.: EVA. 
Negt, Oskar/Kluge, Alexander (1972): Öffentlichkeit und Erfahrung, Frankfurt/M.: Suhrkamp. - (1992): Maßverhältnisse des Politischen, Frankfurt/M.: Fischer.

Nowak, Jörg (2009): Geschlechterpolitik und Klassenherrschaft, Münster: Westf. Dampfboot.

Opratko, Benjamin (2014): Hegemonie, Münster: Westf. Dampfboot.

Peter, Lothar (2010): Was machen wir mit dem Klassenbegriff?, in: Z-Zeitschriftfür marxistische Erneuerung, 21. Jg., H. 81: 133-148.

- (2010): Antwort auf die kritischen Äußerungen zu meinem Artikel „Was machen wir mit dem Klassenbegriff?", in: Z - Zeitschrift für marxistische Erneuerung, 21.Jg., H. 84: 163-160.

Pijl, Kees van der (1984): The Making of an Atlantic Ruling Class, London: Verso.

Poulantzas, Nicos (1968/1974): Politische Macht und gesellschaftliche Klassen, Frankfurt/M.: Athenäum Fischer.

- (1974): Klassen im Kapitalismus - heute, Westberlin: VSA.

Projekt Klassenanalyse (1974): Materialien zur Klassenstruktur der BRD. Erster Teil: Theoretische Grundlagen und Kritik, Westberlin 1973: VSA; Zweiter Teil: Grundriss der Klassenverhältnisse (1950-1970), Westberlin 1974: VSA.

Rademacher, Claudia/Wiechens, Peter (Hrsg.) (2001): Geschlecht-Ethnizität-Klasse, Opladen: Leske + Budrich.

Ritsert, Jürgen (1998): Soziale Klassen, Münster: Westfälisches Dampfboot.

Röttger, Bernd (2009): Klassen - Staat - Emanzipation, in: Bescherer/Schierhorn (2009): 89-96. - (2011): Das Landnahmetheorem; eine kritische Würdigung, in: PROKLA, 41. Jg., H. 165: 645-652.

Rosenbaum, Heidi (1992): Proletarische Familien, Frankfurt/M.: Suhrkamp.

Roth, Karl-Heinz (1974): Die andere Arbeiterbewegung, München: Trikont.

- (Hrsg.) (1994): Die Wiederkehr der Proletarität, Köln: Neuer ISP Verlag.

Roth, Karl-Heinz/van der Linden, Marcel (2009): Ergebnisse und Perspektiven, in: van der Linden/ Roth (2009): 557-600.

Schulze, Gerhard (1992): Die Erlebnisgesellschaft. Kultursoziologie der Gegenwart, Frankfurt/M.: Campus.

Seibert, Thomas (2012): Humanismus nach dem Tod des Menschen. Flucht und Rückkehr des subjektiven Faktors der Geschichte, in: PROKLA, 42.Jg., Heft 167.305-325.

Silver, Beverly (2003/2005): Forces of Labor. Arbeiterbewegungen und Globalisierung seit 1870, Berlin/Hamburg: Assoziation A.

Smykalla, Sandra/Vinz, Dagmar (Hrsg.) (2011): Intersektionalität zwischen Gender und Diversity (Forum Frauen- und Geschlechterverhältnisse Bd. 30), Münster: Westf. Dampfboot.

Stricker, Ruwen/Tulatz, Kaja (2014): Immer Ärger mit der Multitude, in: Baumann, Claus/ Müller, Jan/Stricker, Ruwen (Hrsg.): Philosophie der Praxis und die Praxis der Philosophie, Münster: Westf. Dampfboot.

Thien, Hans-Günter (2010): Die verlorene Klasse. ArbeiterInnen in Deutschland, Münster: Westf. Dampfboot.

- (Hrsg.) (2011): Klassen im Postfordismus, 2. korr. Aufl., Münster: Westf. Dampfboot.

- (2013): Von der Sozialpartnerschaft zu neuen Konflikten? Die Arbeiterbewegung zwischen 1980 und 2013, in: Technoseum. Landesmuseum für Technik und Arbeit (Hrsg.): Durch Nachtzum Licht? Geschichte der Arbeiterbewegung 1863-2013, Mannheim: 341-367.

Thompson, Edward P. (1980): Plebeische Kultur und moralische Ökonomie, Berlin: Ullstein.

- (1968): The Making of the English Working Class, London: Penguin.

- (1980): Das Elend der Theorie, Frankfurt/New York: Campus.

Tomba, Massimiliano/Bellofiore, Riccardo (2009): Lesarten des Maschinenfragments. Perspektiven und Grenzen des operaistischen Ansatzes und der operaistischen Auseinandersetzung mit Marx, in: van der Linden, Marcel/Roth (2009): 407-431. 
van der Linden, Marcel (2008): Workers oft the World, Leiden: Brill.

van der Linden, Marcel/Roth, Karl-Heinz (Hrsg.) (2009): Über Marx hinaus. Arbeitsgeschichte und Arbeitsbegriff in der Konfrontation mit den globalen Arbeitsverbältnissen des 21. Jabrhunderts, Hamburg/Berlin: Assoziation A.

Vester, Michael/Oertzen, Peter v./Geiling, Heiko/Hermann, Thomas/Müller, Dagmar (2001): Soziale Milieus im gesellschaftlichen Strukturwandel, Frankfurt/M.: Suhrkamp.

Wehler, Hans-Ulrich (2008): Deutsche Gesellschaftsgeschichte, 5. Bd.: Bundesrepublik und DDR 1949-1990, München: Beck.

- (2013): Die Explosion der Ungleichheit, in: Blätter für deutsche und internationale Politik, 22.04.2013.

WerkstattGeschichte (2005): „Klasse“, 41. Jg., H. 41.

Wienold, Hanns (2011): Die Gegenwart der Bourgeoisie. Umrisse einer Klasse, in: Thien (2011): 235-286.

\section{Verlag Westfälisches Dampfioot}

\section{2. korr. Auflage \\ Hans-Günter Thien (Hrsg.) Klassen im Postfordismus 2. korr. Auflage 2011 - 381 Seiten - $€ 29,90$ ISBN: 978-3-89691-781-2}

„Der insgesamt gelungene Sammelband bietet einen gerafften Überblick über die aktuelle Debatte über Klassen vor allem im deutschsprachigen Raum, der ältere Beiträge, eher konventionelle Ansätze und innovative Vorstöße - sowohl in konzeptioneller wie empirischer Hinsicht - vereint." Dieter Boris in: „Z"

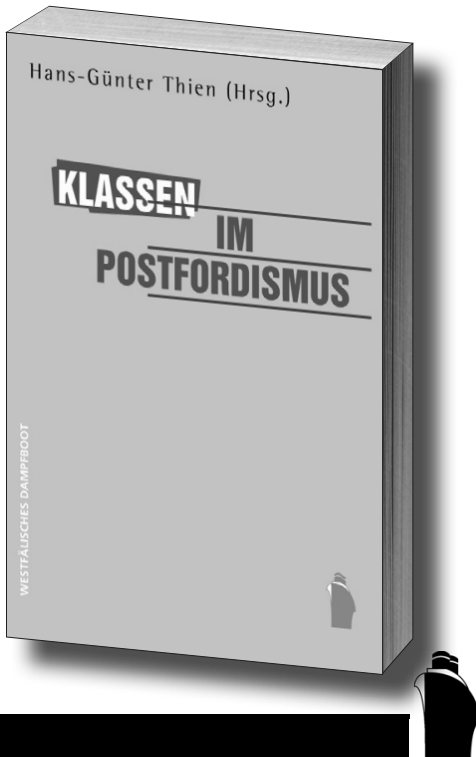

